\title{
Origin of static friction and its relationship to adhesion at the atomic scale
}

\author{
Qing Zhang, ${ }^{1}$ Yue Qi, ${ }^{2}$ Louis G. Hector, Jr., ${ }^{2}$ Tahir Cagin, ${ }^{1}$ and William A. Goddard III ${ }^{1}$ \\ ${ }^{1}$ Materials and Process Simulation Center, 139-74, California Institute of Technology, Pasadena, California 91125, USA \\ ${ }^{2}$ GM Research \& Development Center, Warren, Michigan 48090, USA
}

(Received 19 June 2006; revised manuscript received 27 November 2006; published 27 April 2007)

\begin{abstract}
Using atomic scale interfaces consisting of slabs of the same materials, we study the relationship between adhesion and static friction. The work of separation, which is a measure of adhesion, and the spatial variation of the interface potential energy along the sliding direction are computed for both commensurate and incommensurate $\mathrm{Al}_{2} \mathrm{O}_{3} / \mathrm{Al}_{2} \mathrm{O}_{3}$ interfaces, and incommensurate smooth and rough $\mathrm{Al} / \mathrm{Al}$ interfaces. These values are compared with the predicted static friction stress resulting from constant force and constant velocity molecular dynamics simulations. Simulation results show that static friction is not determined by the absolute value of adhesion. Rather, it is determined by the change of potential energy along the sliding direction.
\end{abstract}

DOI: $10.1103 /$ PhysRevB.75.144114

PACS number(s): 62.20.Qp, 46.55.+d, 81.40.Pq, 68.35.Np

\section{INTRODUCTION}

The relationship between adhesion and friction has long been a topic of significant interest in tribology. In macroscopic experiments, for example, it has generally been observed that larger adhesion leads to higher friction. This observation, which has recently found support from modern surface measurement instrumentation, such as the atomic force microscope, ${ }^{1}$ can be understood with a continuum model. If two surfaces adhere in the absence of an externally applied normal load, the adhesive force between them acts as an effective load resulting in a finite friction force. ${ }^{2}$ Indeed, more than five decades ago, Bowden and Tabor suggested that the laws of adhesion closely resemble those of friction, and demonstrated that for lightly loaded metallic contacts, adhesion increases monotonically with friction coefficient. ${ }^{2}$

Interestingly, there are cases where high friction is associated with low adhesion forces. ${ }^{3,4}$ For example, high frictional forces found at two molecularly smooth mica surfaces separated by one or two molecular layers of OMCTS (octamethylcyclotetrasiloxane, a silicon liquid) with low adhesion energy, were attributed to liquid layer solidification under confinement between the two solid surfaces. ${ }^{3,4}$ On the other hand, although the two mica surfaces in humid air showed very low friction, the adhesion energy was very high due to capillarity. ${ }^{3,4}$ This behavior suggests that the relationship between adhesion and friction forces is substantially more complex, making it an important research frontier in tribology.

The static friction force $F_{s}$ is the minimum lateral force to initiate sliding, and the kinetic friction $F_{k}$ (typically $F_{k}<F_{s}$ ) is the force required to maintain sliding. Each exhibits its own relationship to adhesion. Yoshizawa et al. ${ }^{5}$ linked kinetic friction with adhesion energy hysteresis, which is the difference between the work gain due to interface formation, and the work required to separate the interface into its original surfaces. In their work, various monolayer-coated surfaces were used to sample solidlike, liquidlike, and amorphous surfaces. Although these surfaces showed dramatically different sliding behaviors, the average kinetic friction force did not depend on the adhesion energy, but rather on the adhesion energy hysteresis. This relationship was linked to
Tomlinson's energy dissipation model, wherein friction arises from sliding-induced lattice vibrations, and is eventually dissipated as heat. ${ }^{6}$ Hence, kinetic friction is not directly related to adhesion energy or interfacial bonding.

The existence of static friction indicates that an interface is trapped in a potential well. A minimum force must therefore be applied to overcome the potential energy barrier along the sliding path (parallel to the interface). For a commensurate interface, static friction is finite and, unlike kinetic friction, it is not associated with either energy dissipation or sample heating. ${ }^{7}$ For an incommensurate interface within the context of Tomlinson's model, both kinetic and static friction occur in a system that exhibits multistabilities; this implies that there is more than one stable state at the interface. In such a system, surface atoms are treated as balls on springs attached to the bulk material. If atoms have weaker elastic springs than the maximum slope of the interfacial interaction potential, then the sudden surface atom "pops" between multistable states induce energy dissipation even when the mean velocity is zero, leading to finite static friction. ${ }^{6}$ Alternatively, when the elastic compliance of the atoms is much stronger than the interfacial potential energy, the atoms are confined to their lattice sites, resulting in vanishingly small static and kinetic friction. ${ }^{8}$

To explain the universal occurrence of static friction, recent theoretical models have attributed the origin of static friction to weakly adsorbed mobile molecules, ${ }^{9-12}$ glassy interface films, ${ }^{13}$ material transfer, elastic deformation,,${ }^{14}$ and surface roughness. ${ }^{15}$ However, the atomic-scale origin of static friction is still an open question. Furthermore, little attention has been paid to the relationship between static friction and adhesion at the atomic scale. It is unclear if static friction, or the potential well trapping an interface, is indeed related to the work of separation (or ideal adhesion energy), which is defined as the energy required to separate the constituent slabs normally to the interface ignoring plastic and diffusional degrees of freedom.

In the present paper, we use molecular dynamics (MD) to explore the relationship between static friction and adhesion under dry contact at two very different (solidsolid) interfaces, namely, $\mathrm{Al} / \mathrm{Al}$ and $\mathrm{Al}_{2} \mathrm{O}_{3} / \mathrm{Al}_{2} \mathrm{O}_{3}$ (note that $\mathrm{Al}_{2} \mathrm{O}_{3}$ is the same as $\alpha-\mathrm{Al}_{2} \mathrm{O}_{3}$ or the corundum form of aluminum oxide ${ }^{16}$ ). The same systems were previously 
used to study the velocity dependence of kinetic friction. ${ }^{17}$ In addition, the roles of interface commensuration and roughness on static friction are also investigated. Although there are macroscopic experiments in which static friction at $\mathrm{Al}_{2} \mathrm{O}_{3} / \mathrm{Al}_{2} \mathrm{O}_{3}{ }^{18,19}$ and $\mathrm{Al} / \mathrm{Al}^{20}$ has been measured, the absence of absolute static friction measurements for singlecrystal materials precludes a meaningful comparison of our theoretical predictions with these experiments. There is some experimental work addressing the relationship between kinetic friction and adhesion. ${ }^{5}$ To the best of our knowledge, there is no experimental work that discusses the relationship between adhesion and static friction. With MD simulations, however, we can predict meaningful values of adhesion and friction for these well-defined materials. We shall consider only the dependence of static friction on adhesion, but not adhesion hysteresis. This is because adhesion energy hysteresis, defined as the energy difference between interface formation and separation, ${ }^{5}$ is one energy dissipation mechanism related primarily to kinetic friction rather than static friction.

The remainder of this paper is organized as follows. In Sec. II, the reactive force fields used to model bond interactions in $\mathrm{Al}$ and $\mathrm{Al}_{2} \mathrm{O}_{3}$ as well as related MD simulation details are discussed. The relationship between adhesion, static friction and interfacial energy is explored in Sec. III via a discussion of the MD simulation results. The main results of the work are summarized in Sec. IV.

\section{SIMULATION DETAILS}

The recently developed REAXFF (reactive force fields) ${ }^{21}$ for $\mathrm{Al}_{2} \mathrm{O}_{3}$ and $\mathrm{Al}^{22}$ were used in the MD simulations. REAXFF uses fully transferrable parameters derived from quantum mechanics calculations for $\mathrm{Al}$ in metallically bonded $\mathrm{Al}$ metal and ionically bonded $\mathrm{Al}_{2} \mathrm{O}_{3}$. Four interfaces, namely, commensurate and incommensurate $\mathrm{Al}_{2} \mathrm{O}_{3}$ and flat and rough incommensurate $\mathrm{Al} / \mathrm{Al}$, were constructed by bringing two slabs together in the same cell. The slab for the commensurate $\mathrm{Al}_{2} \mathrm{O}_{3}$ interface was constructed as a $4 a \times 4 a \times c$ superlattice with 480 atoms. The lattice constants are $a$ and $c$, where $a=4.78 \AA$ and $c=13.25 \AA .22$ Since each slab in the cell has an integer stoichiometry, there are $384 \mathrm{Al}$ and $576 \mathrm{O}$ atoms in the simulation cell, giving 960 atoms in the interface model. The sliding direction was along the [10 $\overline{1} 0]$ of the slabs. An incommensurate $\mathrm{Al}_{2} \mathrm{O}_{3} / \mathrm{Al}_{2} \mathrm{O}_{3}$ interface was constructed by rotating the top slab $30^{\circ}$ relative to the bottom slab. The sliding direction was the [10 $\overline{10}]$ of the bottom slab and $[11 \overline{2} 0]$ for the upper slab. To minimize lattice mismatch, a $5 a \times 5 a$ dimension for the lower slab was chosen to match the $3 \sqrt{3} a \times 3 \sqrt{3} a$ length of the upper slab, leading to a lattice mismatch of $3.8 \%$; this had no impact on the MD simulation results. Each $\mathrm{Al}_{2} \mathrm{O}_{3}$ slab was $\mathrm{Al}$ terminated (the oxygen termination is unstable). For incommensurate $\mathrm{Al}(001) / \mathrm{Al}(001)$, the [110] axis of the top slab was set to match the [100] direction of the bottom slab, leading to a $45^{\circ}$ mismatch. The bottom slab was a $5 a \times 5 a \times 4 a$ superlattice with $400 \mathrm{Al}$ atoms; the top slab was a $7 \sqrt{2} a \times 7 \sqrt{2} a \times 4 a$ superlattice with $392 \mathrm{Al}$ atoms, leading to a lattice mismatch of $1 \%$ ( $a$ $=4.01 \AA$ for $\mathrm{Al}$ ). Rough incommensurate $\mathrm{Al} / \mathrm{Al}$ was con- structed by randomly deleting about $15 \%$ of the atoms in the two interfacial layers so as to avoid asperity formation. For each slab, 15 atoms were deleted (i.e., eight of 50 atoms from the top layer of the bottom slab and seven of 49 atoms from the bottom layer of the top slab), giving a rms roughness of about $0.6 \AA$. After equilibrating at $300 \mathrm{~K}$ for $5 \mathrm{ps}$, no surface reconstruction was noted. The $x$ direction, which is [100] and [110] for the bottom and the top slabs, respectively, was set as the sliding direction. The interface models were constructed by bringing two slabs into contact. Periodic boundary conditions were applied in the $x-y$ plane and a large vacuum was added along the $z$ direction. The dimensions for commensurate $\mathrm{Al}_{2} \mathrm{O}_{3} / \mathrm{Al}_{2} \mathrm{O}_{3}$, and incommensurate $\mathrm{Al}_{2} \mathrm{O}_{3} / \mathrm{Al}_{2} \mathrm{O}_{3}$, were $19.13 \times 19.13 \times 80 \AA^{3}$ and 24.38 $\times 24.38 \times 80 \AA^{3}$, respectively. Smooth and rough $\mathrm{Al} / \mathrm{Al}$ interface cells were $20.05 \times 20.05 \times 80 \AA^{3}$. Although the dimensions of the simulation models were relatively small, the simulation results were not influenced by model size. For $\mathrm{Al} / \mathrm{Al}_{2} \mathrm{O}_{3}$ REAXFF, there are four energy terms: bond, overcoordination, van der Waals, and Coulomb energy. The bond energy and overcoordination energy are short-range terms. The cutoff radius for the van der Waals and the Coulomb energies were chosen to be $7.5 \AA$, which is smaller than onehalf the size of the simulation model. We also checked the size effect with a simulation cell that was double the above commensurate $\mathrm{Al}_{2} \mathrm{O}_{3} / \mathrm{Al}_{2} \mathrm{O}_{3}$ model along the $x$ direction. The simulations lead to the same critical static friction as the smaller cell. Additional details pertaining to the force field and simulation models can be found in Refs. 21 and 22 .

Molecular dynamics simulations were conducted on each interface model with the constant volume constant energy $(N V E)$ method at an initial temperature of $300 \mathrm{~K}$. The $N V E$ method was chosen to allow both the temperature and stress of the system to change under the external force. The fully relaxed interface structures were subjected to a pair of equal and opposite externally applied forces of magnitude $F_{\text {ex }}$. At each time step, $F_{\text {ex }}$ was applied to every atom in the outmost layers of the top slab, and $-F_{\text {ex }}$ was applied to every atom in the outermost layers of the bottom slab. The outmost layers consisted of one layer of $\mathrm{Al}$ plus one layer of $\mathrm{O}$ in $\mathrm{Al}_{2} \mathrm{O}_{3}$ and one layer in Al. Consequently, all of the atoms in each slab followed the motion of the outmost layer due to $F_{\text {ex }}$. So the applied lateral force was reported as $F_{\text {ex }}$ times the number of atoms in the outmost layers and then divided by the number of atoms in each slab. Molecular dynamics simulations under various applied forces at $0.05 \mathrm{pN} /$ atom increments were conducted, and the time evolution of the sliding distance, measured as the distance between the center of mass of the top and bottom slabs, was recorded.

\section{RESULTS AND DISCUSSION}

\section{A. Critical shear stress}

The evolution of the sliding distance due to selected external forces is shown in Fig. 1(a) for commensurate $\mathrm{Al}_{2} \mathrm{O}_{3} / \mathrm{Al}_{2} \mathrm{O}_{3}$. Two distinct sliding behaviors are suggested, i.e., no sliding and steady state sliding. If the elastic restoring force due to the "springiness" of interfacial atoms exceeds the applied force, or $F_{\mathrm{ex}} \leqslant 5.25 \mathrm{pN} /$ atom (corresponding to 

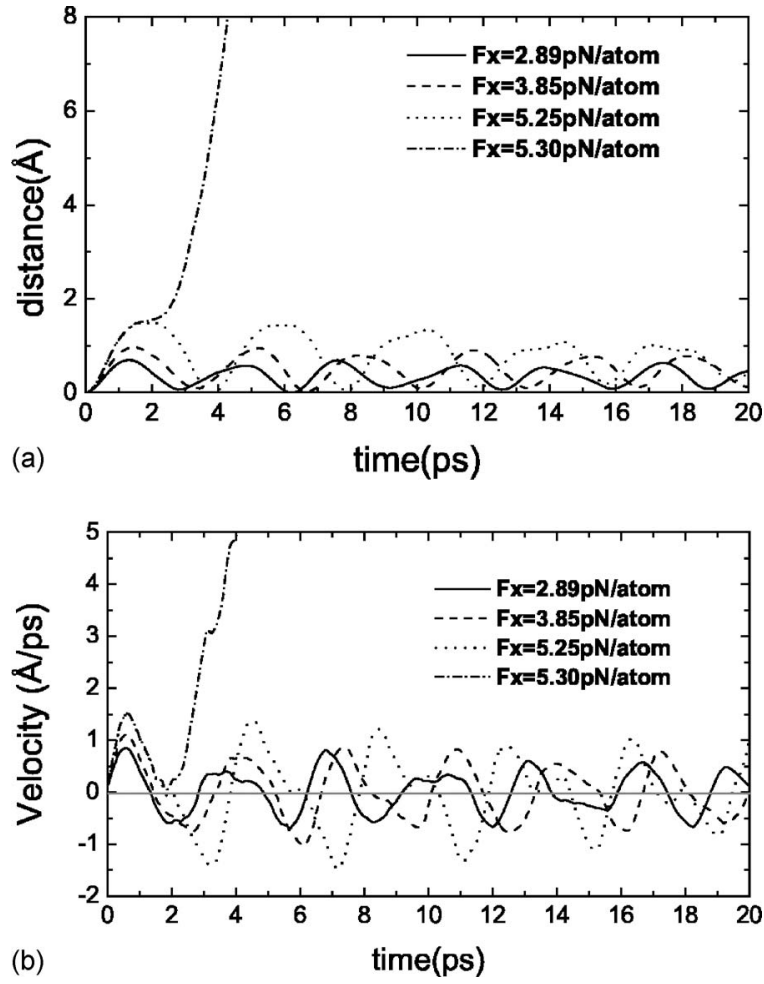

FIG. 1. (a) Distance and (b) velocity as a function of sliding time with different external forces for commensurate $\mathrm{Al}_{2} \mathrm{O}_{3} / \mathrm{Al}_{2} \mathrm{O}_{3}$. The static friction range is $5.25-5.30 \mathrm{pN} /$ atom.

$2.52 \mathrm{nN}$ for the top slab), then the two slabs oscillate with respect to one another about their equilibrium positions: no sliding occurs. This is depicted by the low-amplitude oscillatory curves in Fig. 1(a). If $F_{\mathrm{ex}}>F_{s}$, then the lateral displacement between the two slabs continuously increases, suggesting that the two slabs start sliding. This occurs for $F_{\text {ex }}=5.30$ pN/atom and is denoted by the single curve in Fig. 1(a) that rises to the largest distance shortly after 2 ps. Thus, the range of static friction is $5.25 \leqslant F_{s} \leqslant 5.30 \mathrm{pN} /$ atom $\left(2.52 \leqslant F_{s} \leqslant 2.544 \mathrm{nN}\right.$ for the entire top slab), where $5.25 \mathrm{pN} /$ atom is the largest force without sliding and $5.30 \mathrm{pN} /$ atom is the smallest force with sliding. Similar sliding behaviors were also noted in Ref. 15 at Ni/Ni interfaces. Figure 1(b) also shows the slab velocity under the applied force, (discussed in greater detail in Sec. III C) which shows behavior similar to that in Fig. 1(a). For $F_{\text {ex }} \leqslant 5.25$ pN/atom, there is no heating of the system, while for $F_{\text {ex }}>5.30 \mathrm{pN} /$ atom, both the slab velocity and temperature increase linearly. Because the objective of this paper is to determine the critical force to initiate sliding, the simulations were terminated just after sliding began and the temperature of the system did not, as a consequence, exceed $400 \mathrm{~K}$ within the simulation time of 4 ps.

According to Bowden and Tabor's original adhesion theory, ${ }^{2}$ the friction force at low loads can be written as the critical shear stress (or stress required to separate the interface in shear) $\tau$ and the interface area $A$ as $F_{s}=\tau A$. Considering the different materials and different simulation cell dimensions, the friction forces were normalized by the interface area to compute $\tau$ to facilitate comparison of the different systems. Since the four models all involve singlecrystal materials and the surfaces have no asperities, the interface area was calculated by multiplying the corresponding cell dimensions along the $x$ and $y$ directions. For the rough $\mathrm{Al} / \mathrm{Al}$ surface, atoms were removed on the outmost layer of each slab, so the contact area was similar to that of the flat surface. The critical shear stresses computed with the constant force method are listed in Table I. To initiate sliding for commensurate $\mathrm{Al}_{2} \mathrm{O}_{3} / \mathrm{Al}_{2} \mathrm{O}_{3}$, we found $0.79 \leqslant \tau \leqslant 0.80 \mathrm{GPa}$ $\left(2.52<F_{s}<2.54 \mathrm{nN}\right.$ for total slab). However, for incommensurate $\mathrm{Al}_{2} \mathrm{O}_{3} / \mathrm{Al}_{2} \mathrm{O}_{3}$, a nonvanishing critical shear stress is predicted over the fairly small range $0.13 \leqslant \tau \leqslant 0.14 \mathrm{GPa}$ $\left(0.67<F_{s}<0.72 \mathrm{nN}\right.$ for the total slab), which is clearly smaller than that of the corresponding commensurate interface. Flat, incommensurate $\mathrm{Al}(001) / \mathrm{Al}(001)$ exhibited negligible static friction since $\tau \leqslant 0.003 \mathrm{GPa}\left(F_{s}<0.01 \mathrm{nN}\right.$ for the total slab) to initiate sliding. However, for rough $\mathrm{Al}(001) /$ $\mathrm{Al}(001), 0.17 \leqslant \tau \leqslant 0.19 \mathrm{GPa}\left(0.68<F_{s}<0.76 \mathrm{nN}\right.$ for the total slab), suggesting that roughening at the atomic scale contributes to greater static friction stresses. This observation is in agreement with previous MD simulations for $\mathrm{Ni} / \mathrm{Ni}$ friction. ${ }^{15}$ The static friction must originate from the atomic interaction across the interface, which can be quantified by the interfacial energy. For the three interfaces with nonvanishing static friction, no discontinuous equilibrium positions were observed. Most atoms were very close to their equilibrium positions. As discussed in Ref. 6, the atomic interaction across the interface is too weak compared to the atomic interaction within the bulk material, often referred to as the elastic potential of the bulk material, so multistable states are

TABLE I. Critical shear stress determined with the constant force method, constant velocity method, maximum value of work of separation, $W_{\text {sep }}^{\mathrm{max}}$, and the maximum value of derivative of potential energy for commensurate and incommensurate $\mathrm{Al}_{2} \mathrm{O}_{3} / \mathrm{Al}_{2} \mathrm{O}_{3}$ interfaces, flat and rough incommensurate $\mathrm{Al} / \mathrm{Al}$ interfaces.

\begin{tabular}{|c|c|c|c|c|}
\hline \multirow[b]{2}{*}{ System } & \multicolumn{2}{|c|}{$\tau(\mathrm{GPa})$} & \multirow[b]{2}{*}{$\begin{array}{c}W_{\mathrm{sep}}^{\max } \\
\left(\mathrm{J} / \mathrm{m}^{2}\right)\end{array}$} & \multirow{2}{*}{$\begin{array}{c}\left.\frac{\partial \sigma_{\text {int }}}{\partial x}\right|_{\max } \\
(\mathrm{GPa})\end{array}$} \\
\hline & $\begin{array}{c}\text { From constant force } \\
\text { simulation }\end{array}$ & $\begin{array}{c}\text { From constant velocity } \\
\text { simulation }\end{array}$ & & \\
\hline Commensurate $\mathrm{Al}_{2} \mathrm{O}_{3}$ & $0.79-0.80$ & 1.3 & 0.89 & 1.3 \\
\hline Incommensurate rough $\mathrm{Al}$ & $0.17-0.19$ & 0.4 & 0.45 & 0.4 \\
\hline Incommensurate $\mathrm{Al}_{2} \mathrm{O}_{3}$ & $0.13-0.14$ & 0.4 & 0.18 & 0.42 \\
\hline Incommensurate flat $\mathrm{Al}$ & 0.003 & 0.002 & 0.58 & 0.004 \\
\hline
\end{tabular}


unlikely. For example, the shear elastic constant $\mathrm{C}_{44}$ of $\mathrm{Al}_{2} \mathrm{O}_{3}$ and $\mathrm{Al}$ are 147.6 and $33 \mathrm{GPa}$, respectively; these values are greatly in excess of the critical shear stresses in Table I (calculated from the interfacial potential energy landscape). Therefore, the spring stiffness of the bonds in the top material is much larger than the relative strength of the interface, thereby preventing the surface atoms from jumping between the multistable states (with equal probability) in the potential energy landscape.

\section{B. Adhesion}

Adhesion between two solid surfaces is typically characterized by the work of separation $W_{\text {sep }}$ or the ideal work of adhesion,

$$
W_{\text {sep }}=\sigma_{\text {surf } 1}+\sigma_{\text {surf2 }}-\sigma_{\text {int }}=\left(E_{\text {slab1 }}^{\text {tot }}+E_{\text {slab2 }}^{\text {tot }}-E_{\text {bilayer }}^{\text {tot }}\right) / A .
$$

This is the energy density that is solely due to the breaking of bonds at the interface between the two slabs that comprise the interface such that complete separation results along the interface normal. The interface is assumed to be under vacuum and both materials are assumed to have zero vapor pressures. Note that $\sigma_{\text {surf1 }}$ and $\sigma_{\text {surf2 }}$ are the surface energies and $\sigma_{\text {int }}$ is the interfacial energy density, $E_{\text {slab1 }}^{\text {tot }}$ and $E_{\text {slab2 }}^{\text {tot }}$ are the total energies of slabs 1 and 2, respectively, $E_{\text {bilayer }}^{\text {tot }}$ is the total energy of the interface (consisting of the two slabs), and $A$ is the interfacial area. When the two slabs slide against each other, the total energy of the system will change with the relative positions of the two slabs, due to the atomic configuration change at the interface. Since both the surface energy and the total energy for each slab remain constant during sliding, the interface energy profile $\sigma_{\text {int }}$ varies with $E_{\text {bilayer }}^{\text {tot }}$ and $W_{\text {sep }}$. Therefore, the variation of $W_{\text {sep }}$ with sliding distance from the minimum energy position was calculated and plotted to facilitate comparison of the model interfaces. The initial structures at the outset of sliding resulted from positioning the two slabs relative to one another such that a minimum energy configuration resulted. The top slab was moved to various positions through a lateral spatial increment of $0.2 \AA$ along the sliding direction under the applied force, while the bottom slab was fixed. The direction of relative displacement of the center of mass of the two slabs was defined as the sliding direction. Following each increment of the upper slab, the interface structure was relaxed to a minimum energy configuration, and $W_{\text {sep }}$ was calculated according to Eq. (1). Variation of $W_{\text {sep }}$ along the sliding direction for the four interfaces, namely, commensurate and incommensurate $\mathrm{Al}_{2} \mathrm{O}_{3} / \mathrm{Al}_{2} \mathrm{O}_{3}$ and flat incommensurate and rough incommensurate $\mathrm{Al} / \mathrm{Al}$, is shown in Fig. 2. The maximum value of the work of separation $W_{\text {sep }}^{\max }$ results from the equilibrium interface structure with the minimum potential energy $E_{\text {bilayer }}^{\text {tot }}$ Hence, $W_{\text {sep }}^{\max }$ is defined as the predicted ideal work of adhesion and is listed in Table I. $W_{\text {sep }}^{\max }$ ranges from the largest value of $0.89 \mathrm{~J} / \mathrm{m}^{2}$, associated with commensurate $\mathrm{Al}_{2} \mathrm{O}_{3} / \mathrm{Al}_{2} \mathrm{O}_{3}$, to $0.58 \mathrm{~J} / \mathrm{m}^{2}$ for the flat $\mathrm{Al} / \mathrm{Al}$ interface, to $0.45 \mathrm{~J} / \mathrm{m}^{2}$ for the rough $\mathrm{Al} / \mathrm{Al}$ interface, and finally, to the smallest value of $0.18 \mathrm{~J} / \mathrm{m}^{2}$ for incommensurate $\mathrm{Al}_{2} \mathrm{O}_{3} / \mathrm{Al}_{2} \mathrm{O}_{3}$.

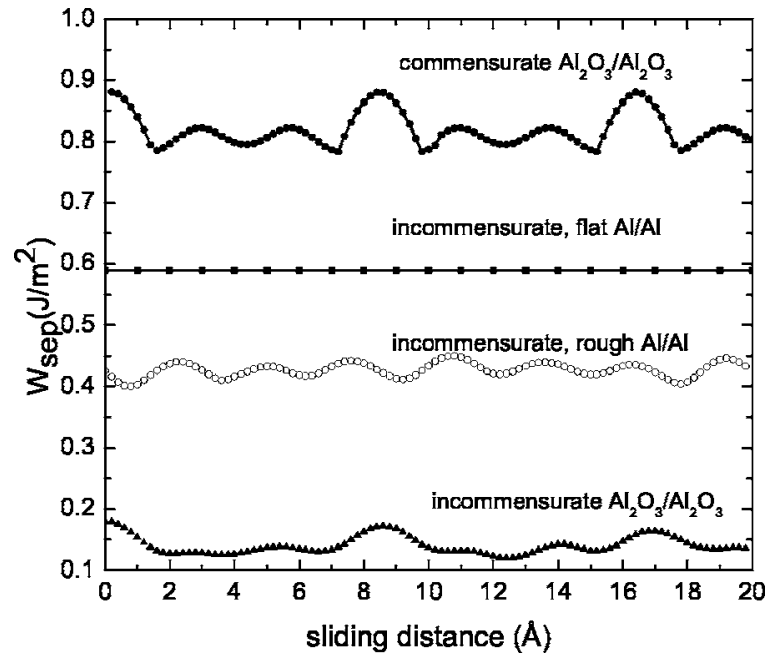

FIG. 2. $W_{\text {sep }}$ along the sliding direction for commensurate (full circles) and incommensurate (triangles) $\mathrm{Al}_{2} \mathrm{O}_{3} / \mathrm{Al}_{2} \mathrm{O}_{3}$ and flat (squares) and rough (open circles) $\mathrm{Al} / \mathrm{Al}$.

\section{Relationship between critical shear stress and adhesion}

For the systems under investigation, the data in Table I suggest that the friction stress level cannot be immediately inferred from $W_{\text {sep }}^{\max }$ (i.e., higher adhesion does not imply higher static friction). This is supported by the observation that the smallest critical shear stress $(0.003 \mathrm{GPa})$ in Table I is predicted for the flat incommensurate $\mathrm{Al} / \mathrm{Al}$ interface, which has the second largest $W_{\text {sep }}$; however, the smallest $W_{\text {sep }}^{\max }$ is associated with the incommensurate $\mathrm{Al}_{2} \mathrm{O}_{3} / \mathrm{Al}_{2} \mathrm{O}_{3}$ interface, which has a larger critical shear stress $(0.17-0.19 \mathrm{GPa})$.

Figure 2 suggests that $W_{\text {sep }}$ fluctuates during sliding for all interfaces except incommensurate, flat $\mathrm{Al} / \mathrm{Al}$. The hills and valleys correspond to the maxima and minima of the interfacial energy density $\sigma_{\text {int }}$ along the sliding direction. The energy barrier is the difference between the maxima and minima. The amplitudes of the interfacial energy change during sliding in the same way as $W_{\text {sep }}$, which also differs for the four interfaces. Therefore, the interfacial energy barrier is the same as the energy barrier noted in $W_{\text {sep }}$. Commensurate $\mathrm{Al}_{2} \mathrm{O}_{3} / \mathrm{Al}_{2} \mathrm{O}_{3}$ has the largest energy barrier, corresponding to the largest $\tau$. Flat incommensurate $\mathrm{Al} / \mathrm{Al}$ has the smallest energy barrier to sliding as shown by the flat curve in Fig. 2, corresponding to a negligible value of $\tau$. The imposed roughness at the $\mathrm{Al} / \mathrm{Al}$ interface increased the energy barrier dramatically compared to that associated with the smooth $\mathrm{Al} / \mathrm{Al}$ interface, leading to an increase in $\tau$. Therefore, it is the amplitude of the change in interfacial energy that correlates with the magnitude of $\tau$. When the interface is trapped in a minimum energy configuration, the applied force $F_{\text {ex }}$ must be larger than a threshold value to overcome the interfacial energy barrier to sliding. Therefore the static friction (critical shear stress) is the force (stress) needed to overcome the energy barrier during sliding. This threshold force is associated with a nonzero static friction or $\tau$. Hence, it is the relationship between the energy barrier along the interface (rather than $W_{\text {sep }}^{\max }$ ) and the threshold force for sliding that determines the static friction range. 

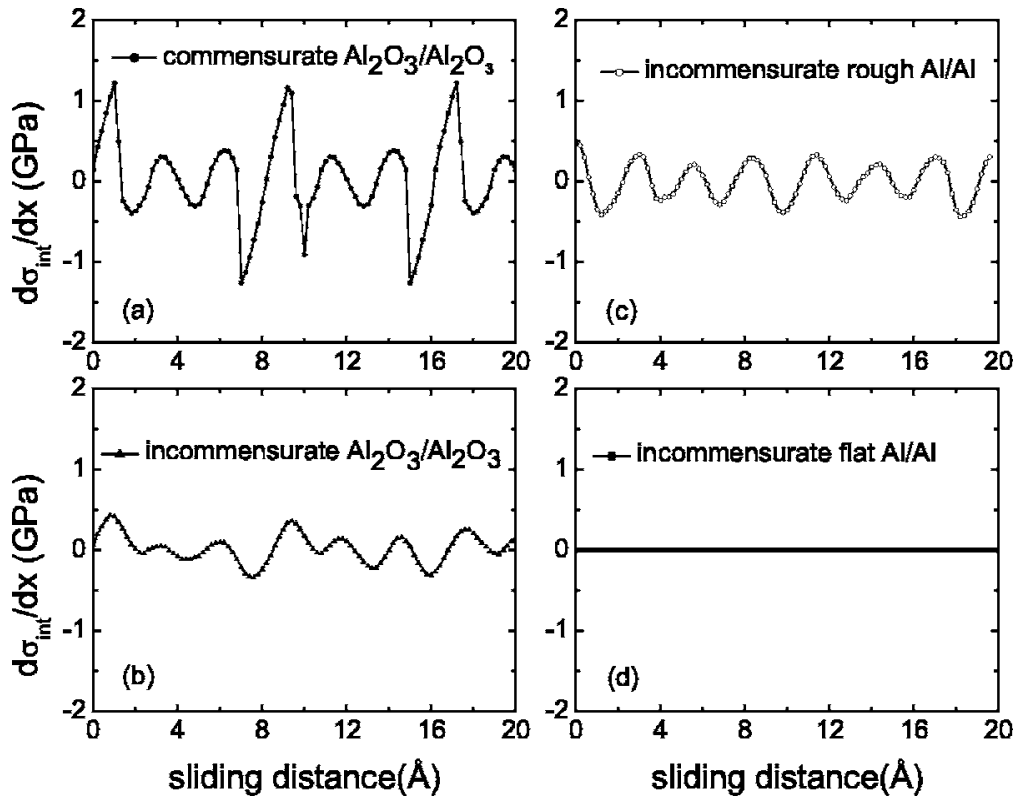

FIG. 3. Variation of $\partial \sigma_{\text {int }} / \partial x$ along the sliding direction for (a) commensurate (full circles), (b) incommensurate (triangles) $\mathrm{Al}_{2} \mathrm{O}_{3} / \mathrm{Al}_{2} \mathrm{O}_{3}$, (c) rough (open circle), and (d) flat (square) $\mathrm{Al} / \mathrm{Al}$.
Early (purely geometric) friction theories suggested that if two slabs are interlocked by asperities, the minimum force to initiate the sliding must exceed the maximum value of the asperity slopes. ${ }^{7}$ Friction theories based upon energy arguments predicted that the maximum slope of the potential, which is the spatial derivative of interfacial energy with respect to sliding distance, is given by $\tau \cdot{ }^{23}$ From Eq. (1), we have $\partial \sigma_{\text {int }} / \partial x=-\partial W_{\text {sep }} / \partial x$ (recall that the surface and total energies of each slab remain constant during sliding); the spatial variation of $\partial \sigma_{\text {int }} / \partial x$ with sliding distance is shown in Fig. 3 for each interface. The maximum values of the curves in Fig. 3, $\left.\left(\partial \sigma_{\text {int }} / \partial x\right)\right|_{\max }$, are listed in Table I for comparison with the predicted $\tau$ in the current constant force model. For the constant force method, we note that the extreme value of $\left.\left(\partial \sigma_{\text {int }} / \partial x\right)\right|_{\max }$ corresponds to the extreme value of $\tau$ associated with commensurate $\mathrm{Al}_{2} \mathrm{O}_{3} / \mathrm{Al}_{2} \mathrm{O}_{3}$, and the smallest value of $\left.\left(\partial \sigma_{\text {int }} / \partial x\right)\right|_{\max }$ is associated with the incommensurate flat $\mathrm{Al} / \mathrm{Al}$ interface which also has the smallest $\tau$. Intermediate values of $\left.\left(\partial \sigma_{\text {int }} / \partial x\right)\right|_{\max }$ are predicted for the remaining two interface models which have $\tau$ values intermediate to commensurate $\mathrm{Al}_{2} \mathrm{O}_{3} / \mathrm{Al}_{2} \mathrm{O}_{3}$ and incommensurate flat $\mathrm{Al} / \mathrm{Al}$. These observations support the notion that the critical friction stress in shear is sensitive to the spatial gradient of the interface energy instead of $W_{\text {sep }}^{\max }$.

We note that the static friction stress $\tau$ can also be calculated from a constant velocity MD simulation in which one slab is moved at a prescribed velocity, and the instantaneous frictional force can be directly calculated from the forces that develop on the atoms in the slab. In previous studies, MD simulations have been conducted on the same systems with constant velocity ranging from $10-400 \mathrm{~m} / \mathrm{s} .{ }^{17}$ For $\mathrm{Al}_{2} \mathrm{O}_{3} / \mathrm{Al}_{2} \mathrm{O} 3$ interfaces, the instantaneous kinetic friction at relatively low velocities $(10-100 \mathrm{~m} / \mathrm{s})$ showed a stick-slip sliding behavior, and the highest lateral force due to the stick force was defined as the static friction force in the simulations (a definition that is supported by numerous experiments). ${ }^{24}$ The $\tau$ values, i.e., static friction force per contact area, calculated from the constant velocity simulation method are also listed in Table I. It should be noted that velocities in our simulations are much larger than those in experiments due to the short time period associated with molecular dynamics simulations. However, we believe this should not influence the major conclusions of this paper.

We note that the critical shear stress calculated from the constant velocity method, which is the maximum value of the instantaneous kinetic friction, equals the predicted $\left.\left(\partial \sigma_{\text {int }} / \partial x\right)\right|_{\text {max }}$. However, the $\tau$ values from the constant force simulations were smaller, i.e., about one-half of $\left.\left(\partial \sigma_{\text {int }} / \partial x\right)\right|_{\text {max }}$, as indicated in Table I. The reason for the simulated static friction being smaller than the maximum energy slope is related to the inertia effect ${ }^{25}$ of the sliding slabs when overcoming the energy barrier. In the constant velocity simulations, the external force must always equal the friction force to maintain a constant velocity. Therefore, the static friction must equal $\left.\left(\partial \sigma_{\text {int }} / \partial x\right)\right|_{\max }$ to overcome the energy barrier. However, in the constant force simulations, the slab velocity is not constant. Figure 1(b) shows that the velocity changes as a function of sliding time for commensurate $\mathrm{Al}_{2} \mathrm{O}_{3} / \mathrm{Al}_{2} \mathrm{O}_{3}$ with different external forces $F_{\text {ex }}$. Comparison of Figs. 1(a), 1(b), and 3(a), suggests that the two $\mathrm{Al}_{2} \mathrm{O}_{3}$ slabs were initially stuck in a potential well. The net force experienced by the slabs was the difference between the external force $F_{\mathrm{ex}}$ and the frictional force due to the interfacial energy, $f=\partial \sigma_{\text {int }} / \partial x$. Figure 3(a) suggests that $f$ increases from zero to its maximum at a $1 \AA$ sliding distance. Hence, when the net force $F_{\mathrm{ex}}-f$ is initially positive, the slabs moved with increasing velocity. When $F_{\text {ex }}-f$ becomes zero, the velocity reaches its maximum value at about $0.6 \mathrm{ps}$ for each $F_{\text {ex }}$ and the corresponding sliding distances are all less than $1 \AA$ up to this time. However, as Fig. 3(a) suggests, $f$ continued to increase, thereby leading to a negative net force, under which the velocity began to decrease. However, the slabs continued to move forward as long as the velocity was still positive. If the velocity decreased to a negative value, the slabs moved backward to their initial positions or the location with minimum potential energy, where $\partial \sigma_{\text {int }} / \partial x=0$. As shown in Fig. 1(a) for all cases with 
$F_{\text {ex }}<5.30$ pN/atom, both sliding velocity and distance oscillated around the equilibrium position. On the other hand, if the velocity never went negative during sliding, the slabs would keep moving forward, as shown for $F_{\text {ex }}$ $=5.30 \mathrm{pN} /$ atom in Fig. 1(a). Therefore, the required condition for continuous sliding in the constant force simulation is that the velocity be positive. Due to the inertia effect, the slabs can move over the steepest point on the energy profile even when $F_{s}<\left.\left(\partial \sigma_{\text {int }} / \partial x\right)\right|_{\max }$ while the velocity is still positive. For example, taking a cosine function as the potential energy $\sigma_{\text {int }}=\cos (x)$, the static friction force should be larger than the average slope of the cosine function in the range of the ascending part (i.e., from $\pi$ to $2 \pi$ ), which is $\left.\left.\frac{2}{\pi}\left(\partial \sigma_{\mathrm{int}} / \partial x\right)\right|_{\max } \approx 0.6\left(\partial \sigma_{\mathrm{int}} / \partial x\right)\right|_{\max }$, instead of the maximum slope $\left.\left(\partial \sigma_{\text {int }} / \partial x\right)\right|_{\max }$. Therefore, $\left.0.6\left(\partial \sigma_{\text {int }} / \partial x\right)\right|_{\max }$ is quite close to the critical stress determined by the constant force simulation displayed in Table I. In conclusion, $\left.\left(\partial \sigma_{\text {int }} / \partial x\right)\right|_{\max }$ gives the upper bound of the static friction, whereas static friction can be lower than $\left.\left(\partial \sigma_{\text {int }} / \partial x\right)\right|_{\max }$, because of the inertia effect or sliding history. ${ }^{25}$

The last question on the origin of static friction is how the energy barrier arises in the first place. This is an important question since the energy barrier is largely responsible for the atomic origin of static friction. The interfacial energy can be assumed as resulting from the summation of atomic energy on each atom sitting on a periodic lattice with lattice constant of $a$, with a single Fourier component, ${ }^{23}$ i.e.,

$$
\sigma_{\text {int }}=\sum_{i} V_{i}\left(x_{i}\right)=\sum_{i} E_{i 0} \cos \left(\frac{2 \pi x_{i}}{a}\right)
$$

where $V_{i}$ is the atomic energy and $E_{i 0}$ is the amplitude of $V_{i}(x)$. Obviously, if the interface is commensurate, the energies of all the atoms add in phase, giving quite a large energy barrier and a large static friction (as is the case for commensurate $\mathrm{Al}_{2} \mathrm{O}_{3} / \mathrm{Al}_{2} \mathrm{O}_{3}$ ). In an incommensurate interface, if the ratio of the lattice constants of the two surfaces is irrational, then the atoms in the top surface sample all phases of the periodic potential with equal probability. Hence, the sum of $V_{i}(x)$ can be a constant without fluctuation, which leads to zero static friction. ${ }^{23}$ Although under the periodic boundary condition, the top and bottom slabs must have similar lengths in the $x-y$ plane, there is no irrational ratio between the two lattice constants of the respective slabs. If the mismatch is small enough and the number of surface atoms is large enough to sample all phases of the periodic potential, this assumption still holds true for the incommensurate ${ }^{23}$ flat $\mathrm{Al} / \mathrm{Al}$ interfaces. However, the interface energy for incommensurate $\mathrm{Al}_{2} \mathrm{O}_{3} / \mathrm{Al}_{2} \mathrm{O}_{3}$ showed some fluctuations along the sliding direction, which led to a nonvanishing static friction [see Fig. 3(b)]. The fluctuation of the interfacial energy at the $\mathrm{Al}_{2} \mathrm{O}_{3} / \mathrm{Al}_{2} \mathrm{O}_{3}$ interface came mainly from the bonding interaction between interfacial $\mathrm{Al}$ atoms, while the bond interaction from $\mathrm{Al}$ and $\mathrm{O}$ quickly dropped to zero at distances in excess of $3 \AA$. The density of $\mathrm{Al}$ atoms at the $\mathrm{Al}_{2} \mathrm{O}_{3}$ surface was only 0.05 atom $/ \AA^{2}$, about one-half that of the Al slab surface $\left(0.1\right.$ atom $\left./ \AA^{2}\right)$. There were fewer interactions across the $\mathrm{Al}_{2} \mathrm{O}_{3} / \mathrm{Al}_{2} \mathrm{O}_{3}$ interface than across the $\mathrm{Al} / \mathrm{Al}$ interface. Therefore, these interactions cannot cancel each other thereby resulting in a nonconstant total energy profile and nonzero static friction. By simply replacing the $\mathrm{O}$ atoms with $\mathrm{Al}$ atoms in $\mathrm{Al}_{2} \mathrm{O}_{3}$, the density of $\mathrm{Al}$ at the surface would increase to 0.2 atom $/ \AA^{2}$, sufficient to cancel the $\mathrm{Al}-\mathrm{Al}$ interactions across the interface, leading to an almost constant energy profile and a zero static friction.

The surface topography also contributes to the static friction. For the flat incommensurate $\mathrm{Al} / \mathrm{Al}$ interface, the total interfacial energy canceled out and the static friction vanished. At the rough incommensurate $\mathrm{Al} / \mathrm{Al}$ interface, the defects at the interface changed the potential of the atoms dramatically, precluding total cancellation of the atomic interaction across the interface. So the interfacial energy still had barriers and a finite static friction was required to overcome these barriers.

\section{SUMMARY}

In summary, the interfacial energy arises from the atomic interactions across the interface. When two slabs comprising a clean, atomically smooth interface are at a potential energy minimum, a critical lateral force or a shear stress, defined as the static friction stress, is required to initiate sliding. The static friction largely results from the interfacial potential energy barrier during sliding; however, it is not always equal to the maximum spatial gradient of the interfacial potential energy profile along the sliding direction. In a constant velocity simulation, the static friction or critical shear stress equals the maximum spatial gradient of the interfacial potential energy; however, in a constant force simulation, it is about $60 \%$ of this value because of the inertia effect.

Contrary to the belief that larger adhesion leads to higher friction, we find that neither the energy barrier during sliding nor the static friction is directly related to the adhesion energy or the work of separation, which is defined as the energy required to separate the interface normally into free surfaces. Simulation results also showed that increasing surface commensurability and roughness can increase the maximum energy barrier associated with the interfacial potential energy profile leading to increased static friction. Although, the simulations were conducted on similar material interfaces, i.e., $\mathrm{Al} / \mathrm{Al}$ and $\mathrm{Al}_{2} \mathrm{O}_{3} / \mathrm{Al}_{2} \mathrm{O}_{3}$, we believe that the general relationship between energy barrier and static friction is independent of the material systems and is also appropriate for different material interfaces (e.g., $\mathrm{Al} / \mathrm{Al}_{2} \mathrm{O}_{3}$ ), provided there is no asperity contact. The similar material interfaces were simply expedients that allowed us to demonstrate the proposed relationship between friction and adhesion.

\section{ACKNOWLEDGMENTS}

The authors wish to acknowledge Y. T. Cheng and M. H. Müser for useful discussions on the results. Support of the Caltech research was provided through a grant from General Motors. The facilities of MSC are supported by funding from ONR-DURIP, ARO-DURIP, and Beckman Institute with additional funding from NSF, NIH, DoE, ONR, DARPA, Nissan, ChevronTexaco, Dow-Corning, Intel, Aventis Pharma, and Berlex Biopharma. 
${ }^{1}$ Y. Li and D. Y. Li, J. Appl. Phys. 95, 7961 (2004).

${ }^{2}$ F. P. Bowden and D. Tabor, The Friction and Lubrication of Solids, Part II (Oxford University Press, Oxford, 1964).

${ }^{3}$ M. Homola, J. N. Israelachvili, P. M. McGuiggan, and M. L. Gee, Wear 136, 65 (1990).

${ }^{4}$ M. Homola, J. N. Israelachvili, M. L. Gee, and P. M. McGuiggan, J. Tribol. 111, 675 (1989).

${ }^{5}$ H. Yoshizawa, Y. L. Chen, and J. Israelachvili, J. Phys. Chem. 97, 4128 (1993).

${ }^{6}$ G. A. Tomlinson, Philos. Mag. 7, 905 (1929).

${ }^{7}$ J. Krim, Am. J. Phys. 79, 890 (2002).

${ }^{8}$ Caroli and Ph. Nozières, Eur. Phys. J. B 4, 233 (1998).

${ }^{9}$ G. He, M. H. Muser, and M. O. Robbins, Science 284, 1650 (1999).

${ }^{10}$ C. Daly, J. Zhang, and J. B. Sokoloff, Phys. Rev. Lett. 90, 246101 (2003).

${ }^{11}$ M. H. Muser and M. O. Robbins, Phys. Rev. B 61, 2335 (2000).

${ }^{12}$ M. H. Müser, Phys. Rev. Lett. 89, 224301 (2002).

${ }^{13}$ J. B. Sokoloff, Phys. Rev. B 65, 115415 (2002).

${ }^{14}$ M. Muser, Tribol. Lett. 10, 15 (2001).

${ }^{15}$ Y. Qi, Y. T. Cheng, T. Cagin, and William A. Goddard III, Phys.
Rev. B 66, 085420 (2002).

${ }^{16}$ K. Wefers and C. Misra, Alcoa Technical Paper No. 19, revised, 1987 (unpublished).

${ }^{17}$ Q. Zhang, Y. Qi, L. G. Hector, Jr., T. Cagin, and William A. Goddard III, Phys. Rev. B 72, 045406 (2005).

${ }^{18}$ B. Vick, M. J. Furey, and K. Iskanda, Tribol. Trans. 42, 888 (1999).

${ }^{19}$ D. H. Hwang and K. H. Z. Gahr, Wear 255, 365 (2003).

${ }^{20}$ K. Noguchi, H. Fujita, M. Suzuki, and N. Yoshimura, in Proceedings of the Conference on IEEE Microelectromechanical Systems 1991 (IEEE, New York, 1991), 148-153.

${ }^{21}$ A. C. T. van Duin, S. Dasgupta, F. Lorant, and W. A. Goddard III, J. Phys. Chem. A 105, 9396 (2001).

${ }^{22}$ Q. Zhang, T. Cagin, A. van Duin, William A. Goddard III, Y. Qi, and L. G. Hector, Jr., Phys. Rev. B 69, 045423 (2004).

${ }^{23}$ M. O. Robbins and M. H. Muser, in Modern Tribology Handbook, edited by B. Bhushan (CRC Press, Boca Raton, FL, 2001), Vol. 1, pp. 717-765.

${ }^{24}$ M. H. Muser, L. Wenning, and M. O. Robbins, Phys. Rev. Lett. 86, 1295 (2001).

${ }^{25}$ M. Weiss and F. J. Elmer, Phys. Rev. B 53, 7539 (1996). 